\title{
Fulminant Hemorrhagic Bullae of the Upper Extremities Arising in the Setting of IV Placement During Severe COVID-19 Infection: Observations From a Major Consultative Practice
}

\author{
Elena Kurland, MD; Ben J. Friedman, MD; Pranita Rambhatla, MD
}

\section{PRACTICE POINTS}

- Hemorrhagic bullae are an uncommon cutaneous manifestation of COVID-19 infection in hospitalized individuals.

- Although there is no reported treatment for COVID-19-associated hemorrhagic bullae, we recommend supportive care and management of underlying etiology.

\section{To the Editor:}

A range of dermatologic manifestations of COVID-19 have been reported, including nonspecific maculopapular exanthems, urticaria, and varicellalike eruptions. ${ }^{1}$ Additionally, there have been sporadic accounts of cutaneous vasculopathic signs such as perniolike lesions, acro-ischemia, livedo reticularis, and retiform purpura. ${ }^{2}$ We describe exuberant hemorrhagic bullae occurring on the extremities of 2 critically ill patients with COVID-19. We hypothesized that the bullae were vasculopathic in nature and possibly exacerbated by peripheral intravenous (IV)-related injury.

A 62-year-old woman with a history of diabetes mellitus and chronic obstructive pulmonary disease was admitted to the intensive care unit for acute hypoxemic respiratory failure secondary to COVID-19 infection.
Dermatology was consulted for evaluation of blisters on the right arm. A new peripheral IV line was inserted into the patient's right forearm for treatment of secondary methicillin-resistant Staphylococcus aureus pneumonia. The peripheral IV was inserted into the right proximal forearm for 2 days prior to development of ecchymosis and blisters. Intravenous medications included vancomycin, cefepime, methylprednisolone, and famotidine, as well as maintenance fluids (normal saline). Physical examination revealed extensive confluent ecchymoses with overlying tense bullae (Figure 1). Notable laboratory findings included an elevated D-dimer (peak of $8.67 \mu \mathrm{g} / \mathrm{mL}$ fibrinogen-equivalent units [FEUs], reference range $<0.5 \mu \mathrm{g} / \mathrm{mL}$ FEU) and fibrinogen (789 mg/dL, reference range 200-400 mg/dL) levels. Three days later she developed worsening edema of the right arm, accompanied by more extensive bullae formation (Figure 2). Computed tomography of the right arm showed extensive subcutaneous stranding and subcutaneous edema. An orthopedic consultation determined that there was no compartment syndrome, and surgical intervention was not recommended. The patient's course was complicated by multiorgan failure, and she died 18 days after admission.

A 67-year-old man with coronary artery disease, diabetes mellitus, and hemiparesis secondary to stroke was admitted to the intensive care unit due to hypoxemia secondary to COVID-19 pneumonia. Dermatology

From the Henry Ford Hospital, Detroit, Michigan. Drs. Kurland and Friedman are from the Department of Dermatology, and Dr. Rambhatla is from the Department of Pathology and Laboratory Medicine.

The authors report no conflict of interest.

Correspondence: Elena Kurland, MD, 3031 W Grand Blvd, Detroit, MI 48202 (ekurlan1@hfhs.org).

doi:10.12788/cutis.0326 
was consulted for the evaluation of blisters on both arms. The right forearm peripheral IV line was used for 4 days prior to the development of cutaneous symptoms. Intravenous medications included cefepime, famotidine, and methylprednisolone. The left forearm peripheral IV line was in place for 1 day prior to the development of blisters and was used for the infusion of maintenance fluids (lactated Ringer's solution). On the first day of the eruption, small bullae were noted at sites of prior peripheral IV lines (Figure 3). On day 3 of admission, the eruption progressed to larger and more confluent tense bullae with ecchymosis (Figure 4). Additionally, laboratory test results were notable for an elevated $\mathrm{D}$-dimer (peak of $>20.00 \mathrm{ug} / \mathrm{mL} \mathrm{FEU})$ and fibrinogen $(748 \mathrm{mg} / \mathrm{dL})$ levels. Computed tomography of the arms showed extensive

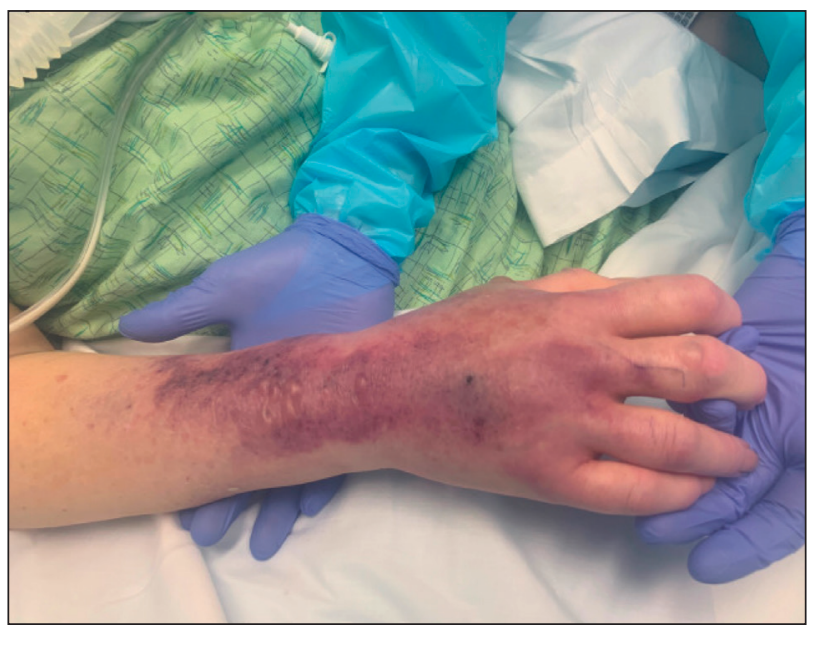

FIGURE 1. Initial presentation of ecchymoses with overlying bullae on the right arm (patient 1).

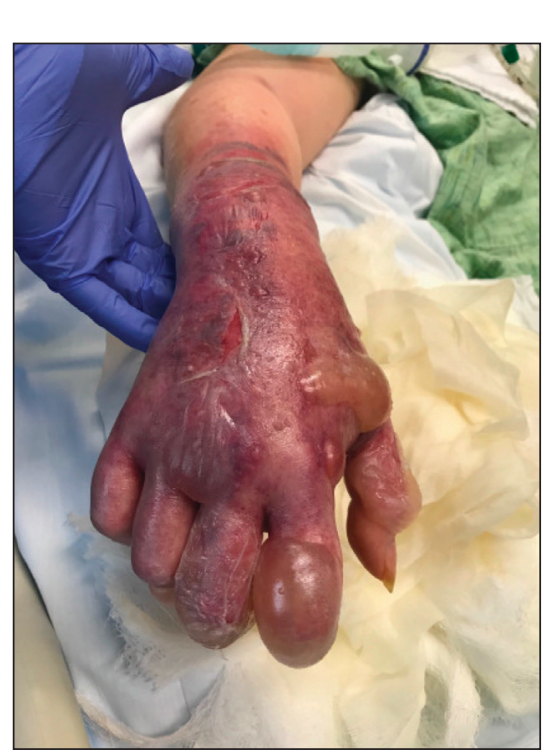

FIGURE 2. Massive hemorrhagic bullae of the right arm 3 days after a peripheral intravenous line was inserted (patient 1). subcutaneous stranding and fluid along the fascial planes of the arms, with no gas or abscess formation. Surgical intervention was not recommended following an orthopedic consultation. The patient's course was complicated by acute kidney injury and rhabdomyolysis; he was later discharged to a skilled nursing facility in stable condition.

Reports from China indicate that approximately $50 \%$ of COVID-19 patients have elevated D-dimer levels and are at risk for thrombosis. ${ }^{3}$ We hypothesize that the exuberant hemorrhagic bullous eruptions in our 2 cases may be mediated in part by a hypercoagulable state secondary to COVID-19 infection combined with IV-related trauma or extravasation injury. However, a direct cytotoxic effect of the virus cannot be entirely excluded as a potential inciting factor. Other entities considered in the differential for localized bullae included trauma-induced bullous pemphigoid as well as bullous cellulitis. Both patients were treated with high-dose steroids as well as

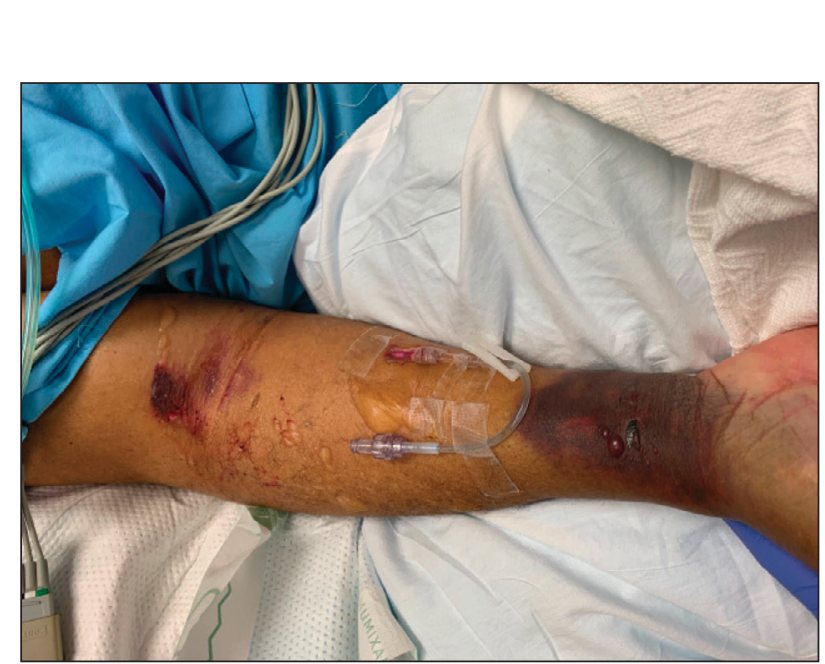

FIGURE 3. Ecchymoses and superficial bullae at the initial presentation at the sites of peripheral intravenous lines (patient 2).

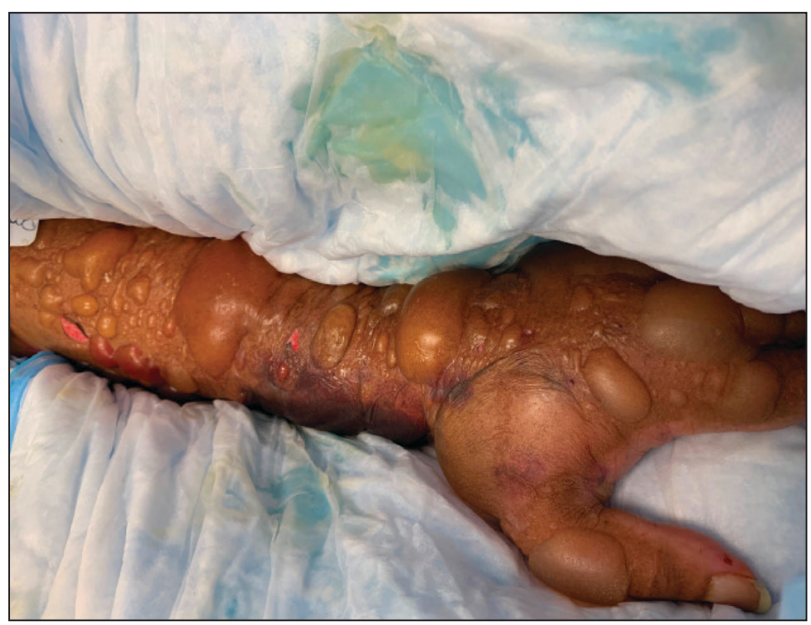

FIGURE 4. Diffuse tense hemorrhagic bullae 3 days after admission (patient 2). 
broad-spectrum antibiotics, which were expected to lead to improvement in symptoms of bullous pemphigoid and cellulitis, respectively; however, they did not lead to symptom improvement.

Extravasation injury results from unintentional administration of potentially vesicant substances into tissues surrounding the intended vascular channel. ${ }^{4}$ The mechanism of action of these injuries is postulated to arise from direct tissue injury from cytotoxic substances, elevated osmotic pressure, and reduced blood supply if vasoconstrictive substances are infused. ${ }^{5}$ In our patients, these injuries also may have promoted vascular occlusion leading to the brisk reaction observed. Although ecchymoses typically are associated with hypocoagulable states, both of our patients were noted to have normal platelet levels throughout hospitalization. Additionally, findings of elevated D-dimer and fibrinogen levels point to a hypercoagulable state. However, there is a possibility of platelet dysfunction leading to the observed cutaneous findings of ecchymoses. Thrombocytopenia is a common finding in patients with COVID-19 and is found to be associated with increased in-hospital mortality. ${ }^{6}$ Additional study of these reactions is needed given the propensity for multiorgan failure and death in patients with COVID-19 from suspected diffuse microvascular damage. ${ }^{3}$

\section{REFERENCES}

1. Recalcati S. Cutaneous manifestations in COVID-19: a first perspective [published online March 26, 2020]. J Eur Acad Dermatol Venereol. doi:10.1111/jdv.16387

2. Zhang $Y$, Cao $W$, Xiao $M$, et al. Clinical and coagulation characteristics of 7 patients with critical COVID-19 pneumonia and acro-ischemia [in Chinese][published online March 28, 2020]. Zhonghua Xue Ye Xue Za Zhi. 2020;41:E006.

3. Mei H, HuY. Characteristics, causes, diagnosis and treatment of coagulation dysfunction in patients with COVID-19 [in Chinese][published online March 14, 2020]. Zhonghua Xue Ye Xue Za Zhi. 2020;41:E002.

4. Sauerland C, Engelking C, Wickham R, et al. Vesicant extravasation part I: mechanisms, pathogenesis, and nursing care to reduce risk. Oncol Nurs Forum. 2006;33:1134-1141.

5. Reynolds PM, MacLaren R, Mueller SW, et al. Management of extravasation injuries: a focused evaluation of noncytotoxic medications. Pharmacotherapy. 2014;34:617-632.

6. Yang $X$, Yang Q, Wang $Y$, et al. Thrombocytopenia and its association with mortality in patients with COVID-19. J Thromb Haemost. 2020;18:1469-1472. 